\title{
Instantaneous Response Patterns of Baroreflex Sensitivity, Respiratory Sinus Arrhythmia Sensitivity and Vagal Activity to Cold Face Test and Active Orthostatic Test
}

\author{
Salvador Carrasco-Sosa ${ }^{1}$, Alejandra Guillén-Mandujano ${ }^{1}$, Aldo R. Mejía-Rodríguez ${ }^{2}$ \\ ${ }^{1}$ División de Ciencias Biológicas y de la Salud, Universidad Autónoma Metropolitana-I, CDMX, \\ México \\ ${ }^{2}$ Universidad Autónoma de San Luis Potosí, San Luis Potosí, México
}

\begin{abstract}
In 25 healthy volunteers, we examined if active orthostatic test (AOT) and cold face test (CFT) cause opposite effects on the instantaneous dynamics of baroreflex sensitivity (BRS), high-frequency power of RR intervals $\left(H_{R R}\right)$, low-frequency power of systolic pressure $\left(L F_{S P}\right), L F_{R R} / H F_{R R}$ ratio and respiratory sinus arrhythmia sensitivity (RSAS), obtained by a timefrequency distribution. We also assessed the correlations between BRS and the spectral measures of autonomic activity. Instantaneous values of $R R, H F_{R R}, B R S$ and RSAS presented characteristic response patterns of overshoot in CFT, and of undershoot of the four variables in AOT. BRS was positively correlated with $H F_{R R}$ (0.85 \pm 0.14$)$, RSAS $(0.76 \pm 0.11)$ and $R R(0.87 \pm 0.11)$, and negatively with $L F_{S P}(-0.81 \pm 0.09)$ and $L F_{R R} / H F_{R R}$ ($0.77 \pm 0.13)$. In a beat-to-beat format BRS, $H F_{R R}, R S A S$, $L F_{S P}$ and $R R$ : exhibit great dynamism, are strongly correlated, and follow distinctive opposite patterns in response to each maneuver. While in CFT the greater values of BRS and RSAS allow amplifying the vagal activity and minimizing the sympathetic outflow, in AOT the lower values of BRS and RSAS determine the opposite autonomic effects. The tight correlations suggest that both BRS and RSAS operate to reciprocally strengthen and inhibit the sympathetic and vagal activities.
\end{abstract}

\section{Introduction}

Cold face test (CFT) and active orthostatic test (AOT) are part of the battery of noninvasive tests commonly used for assessing autonomic-cardiovascular function [1] in physiological and clinical settings. While AOT provokes baroreflex sensitivity (BRS) reduction and shifts the autonomic balance towards sympathetic predominance [2], CFT elicits increases of vagal activity, BRS and respiratory sinus arrhythmia sensitivity (RSAS)
[3]. It has been reported that great fluctuations of arterial pressure (AP) and heart rate (HR) occur in the first minute of AOT [4,5] and CFT [6]. However, the instantaneous time course of BRS has not been studied, the involvement of RSAS is unclear, and a quantitative comparison between their autonomic-cardiovascular effects for normalizing purposes has not been performed yet. For instance, it is unknown how many times larger are the vagal activity, RSAS and BRS changes induced by CFT in relation to those of AOT. We hypothesize that the pooled values of BRS during CFT and AOT will present strong correlations with autonomic indexes, positive with vagal activity measures and negative with the sympathetic outflow ones. Our aims were to examine if AOT and CFT cause opposite effects on the instantaneous dynamics of BRS, RSAS, and sympathovagal balance, and to assess the correlations between the BRS values of the two tests and the spectral measures of autonomic activity, high-frequency power of $\mathrm{RR}$ intervals $\left(\mathrm{HF}_{\mathrm{RR}}\right)$, low-frequency power of systolic pressure $\left(\mathrm{LF}_{\mathrm{SP}}\right), \mathrm{LF}_{\mathrm{RR}} / \mathrm{HF}_{\mathrm{RR}}$ ratio and the RSAS.

\section{Methods}

\subsection{Subjects}

Twenty-five healthy, normotensive and sedentary subjects, 14 men and 11 women, were studied. Mean age, height and weight were $22.2 \pm 2.2$ years, $167 \pm 8 \mathrm{~cm}$ and $69.1 \pm 10.4 \mathrm{~kg}$ respectively. Their written informed consent was requested to participate.

\subsection{Protocol}

Volunteers visited the laboratory twice. The first time, their health status and anthropometric variables were evaluated, and in the second visit the experimental stage was carried out. Volunteers underwent 1-min control, 1min maneuver and 2-min recovery stages for both CFT 
and AOT, applied in random order. To perform AOT, the subjects rapidly stood up in two consecutive movements from the supine position, returning to this position at the end of the maneuver stage. CFT was done by applying a bag filled with ice water at $0^{\circ} \mathrm{C}$ on the face, excluding the eyes, with the subject in supine position. ECG, noninvasive AP, and respiratory volume (Res) signals were recorded during each test.

\subsection{Signal recording and acquisition}

ECG was detected at the CM5 bipolar lead using a bioelectric amplifier (Biopac Systems). AP was measured by Finapres (Ohmeda). Respirogram was obtained by Inductotrace (Ambulatory Monitoring). All signals were digitized at a sampling rate of $1 \mathrm{kHz}$ via an acquisition system (Biopac Systems).

\subsection{Data processing}

R-wave peaks, systolic pressure (SP), diastolic pressure (DP) and Res maxima were detected to construct the R-R intervals (RR), SP, DP, their difference, pulse pressure (PP), tidal volume (TV) and respiratory frequency (RF) time series. These series were cubicspline interpolated, resampled at $4 \mathrm{~Hz}$ and separated into level and oscillations by low-pass filtering. Timefrequency spectra of the oscillations of the series were estimated with the smoothed pseudo-Wigner-Ville distribution and integrated in the standard low frequency (LF) and high frequency (HF) bands to compute the instantaneous values of LF power of SP ( $\left.L F_{S P}\right)$, LF power of RR ( $\left.\mathrm{LF}_{\mathrm{RR}}\right)$, $\mathrm{HF}$ power of $\mathrm{RR}\left(\mathrm{HF}_{\mathrm{RR}}\right), \mathrm{LF}_{\mathrm{RR}} / \mathrm{HF}_{\mathrm{RR}}$ ratio, and $\mathrm{HF}$ power of Res $\left(\mathrm{HF}_{\mathrm{Res}}\right)$. Instantaneous values of $L_{\mathrm{RR}}$ and $L F_{\mathrm{SP}}$ were used to compute BRS by alpha index and their time-frequency coherence. Similarly, instantaneous RSAS was estimated by the square root of the $\mathrm{HF}_{\mathrm{RR}} / \mathrm{HF}_{\mathrm{Res}}$ ratio and their time-frequency coherence. Coherence values greater than 0.5 were considered significant. To highlight any patterned responses, individual indexes dynamics were ensemble-averaged after subtracting their mean baseline value. Additionally, for statistical purposes indexes dynamics were segmented into 6-s epochs.

\subsection{Statistical analysis}

Data are expressed as mean $\pm \mathrm{SD}$. Inter-stage differences were tested by ANOVA for repeated measures. Post-hoc pairwise comparisons were performed by the Tukey test. Inter-maneuver differences were tested by paired t-test. Pooled mean values of the 6-s segments of the indexes dynamics during the two tests were used to compute linear regressions and correlations for each subject. Statistical significance was accepted at $\mathrm{p}<0.05$.

\section{Results}

In the time-frequency spectra of RR and PP series, power depicted a patterned response in each maneuver: undershoot of $\mathrm{HF}_{\mathrm{RR}}$ and overshoot of $\mathrm{LF}_{\mathrm{RR}}$ and $\mathrm{LF}_{\mathrm{PP}}$ in AOT, and the opposite changes of these measures in CFT. $\mathrm{HF}_{\mathrm{RR}}$ power change was greater in CFT than in AOT (Fig. 1). Important fluctuations of the instantaneous power are noticeable in both frequency bands in each maneuver.

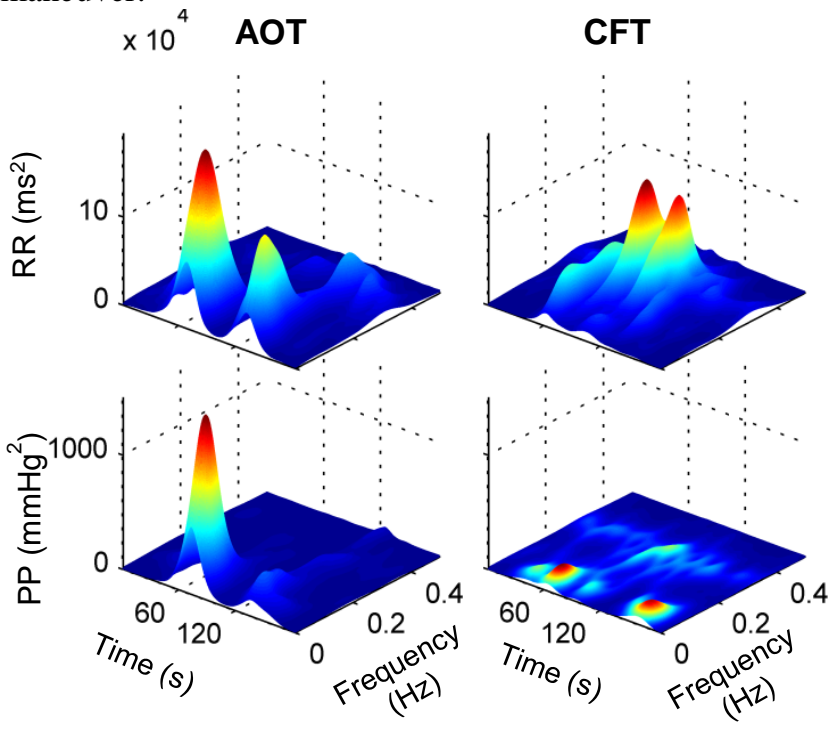

Fig. 1. Representative time-frequency distributions of RR and PP series during AOT (left column) and CFT (right column). Maneuver spans from 60 to $120 \mathrm{~s}$.

Pooled means of PP, RR, lnHFRR, BRS, RSAS, $\mathrm{LF}_{\mathrm{RR}} / \mathrm{HF}_{\mathrm{RR}}, \mathrm{LF}_{\mathrm{SP}}$ (control value $=18.6 \pm 11.4, \mathrm{AOT}=$ 68.9 \pm 30.0 , CFT $=25.1 \pm 21.9$ ), $\mathrm{HF}_{\text {Res }}$ and $\mathrm{TV}$ during the maneuvers were different $(\mathrm{p}<0.03)$ from their baseline values in both AOT and CFT (Fig. 2). The instantaneous values of PP, RR, BRS, $\operatorname{lnHF} F_{R R}$, and RSAS presented similar characteristic response patterns to AOT, consisting of decrease in relation to their baselines, with an abrupt initial fall and an overshoot at the beginning of recovery, with some mid-maneuver differences: PP presented an overshoot and a slight fall at the end (Fig. 2A); RR showed a partial recovery and another fall at the end (Fig. 2B); BRS (mean $\mathrm{LF}_{\mathrm{RR}}-\mathrm{LF}_{\mathrm{SP}}$ coherence= $0.66 \pm 0.11$ ) displayed a gradual recovery (Fig. 2C); the reduction of $\operatorname{lnHF}_{\mathrm{RR}}$ was sustained for the entire maneuver (Fig. 2D); RSAS (mean $\mathrm{HF}_{\mathrm{RR}}-\mathrm{HF}_{\text {Res }}$ coherence $=0.58 \pm 0.10$ ) fell and then recovered partially (Fig. 2E); $\mathrm{HF}_{\mathrm{Res}}$ and $\mathrm{LF}_{\mathrm{RR}} / \mathrm{HF}_{\mathrm{RR}}$ ratio presented similar responses, a large overshoot at the beginning of the maneuver and a smaller one at the end (Fig. 2F-G).

Instantaneous values of PP, RR, BRS (mean $\mathrm{LF}_{\mathrm{RR}}{ }^{-}$ $\mathrm{LF}_{\mathrm{SP}}$ coherence $=0.63 \pm 13$ ), $\operatorname{lnHF} F_{\mathrm{RR}}$, and RSAS (mean $\mathrm{HF}_{\mathrm{RR}}-\mathrm{HF}_{\mathrm{Res}}$ coherence $=0.83 \pm 0.10$ ) also presented patterned responses to $\mathrm{CFT}$, consisting of an increase 
from their baselines, which lasted for most of the maneuver. While the initial increases of $\mathrm{PP}, \operatorname{lnHF}_{\mathrm{RR}}$ and RSAS were abrupt (Fig. 2A, D and E), those of RR and BRS were gradual (Fig. $2 \mathrm{~B}$ and $\mathrm{C}$ ). $\mathrm{LnHF}_{\mathrm{RR}}$ increase preceded that of $\mathrm{BRS}$. $\mathrm{LF}_{\mathrm{RR}} / \mathrm{HF}_{\mathrm{RR}}$ remained below baseline throughout the maneuver (Fig. 2G). AOT and CFT presented slight increases in TV $(p<0.03)$, with differences between maneuvers $(0.71 \pm 0.30$ vs. $0.56 \pm 0.24$ $\mathrm{l}$ respectively, $\mathrm{p}<0.02$ ). During both maneuvers, RF was not different from control or between them ( $R F$ in $\mathrm{AOT}=14.9 \pm 4.6$ vs. in $\mathrm{CFT}=16.5 \pm 3.8$ breaths $/ \mathrm{min})$.

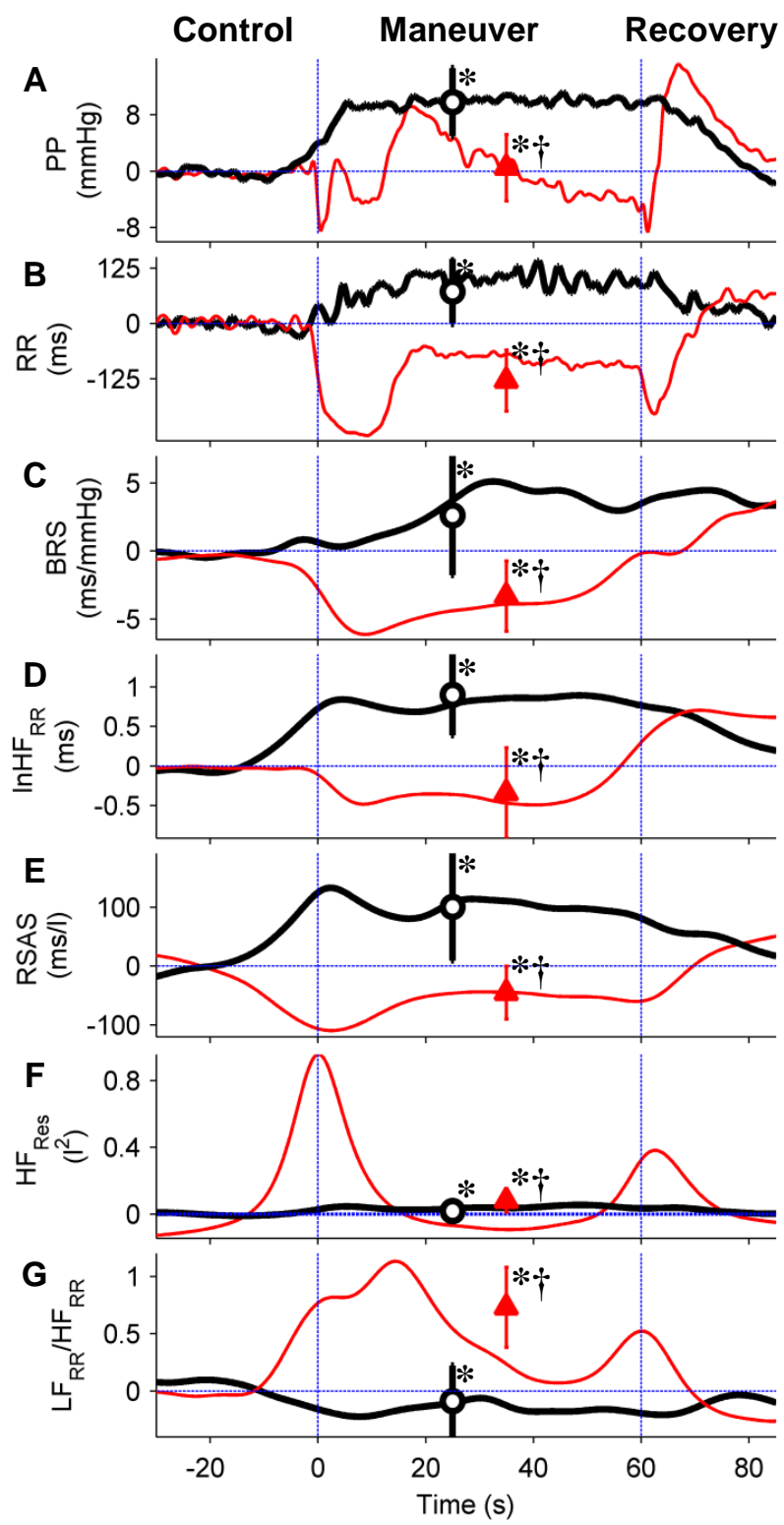

Fig. 2. Ensemble averages of the dynamics of A) PP, B) $\left.\left.\mathrm{RR}, \mathrm{C}) \mathrm{BRS}, \mathrm{D}) \operatorname{lnHF}_{\mathrm{RR}}, \mathrm{D}\right) \mathrm{RSAS}, \mathrm{F}\right) \mathrm{HF}_{\mathrm{Res}}$ and G) $\mathrm{LF}_{\mathrm{RR}} / \mathrm{HF}_{\mathrm{RR}}$ in CFT (thick line) and AOT (thin line), with their pooled means \pm SD (CFT O, AOT $\Delta$ ). ${ }^{*}$ p $<0.01$ maneuver stage vs. baseline, $\uparrow \mathrm{p}<0.001$ CFT vs. AOT.
While pooled means of PP, BRS, $\operatorname{lnHF}_{\mathrm{RR}}$, RSAS and RR were $2.9 \pm 2.3,2.4 \pm 2.3,3.1 \pm 3.0,2.7 \pm 1.3$ and $2.0 \pm 1.4$ times greater $(\mathrm{p}<0.001)$ in CFT than in AOT, those of $\mathrm{HF}_{\mathrm{Res}}, \mathrm{LF}_{\mathrm{RR}} / \mathrm{HF}_{\mathrm{RR}}$ and $\mathrm{LF}_{\mathrm{SP}}$ were $2.5 \pm 1.1,7.2 \pm 6.2$ and $5.1 \pm 5.0$ times smaller $(p<0.001)$. Mean correlations of BRS with $\operatorname{lnHF}_{R R}$ was $0.85 \pm 0.14(\mathrm{p}<0.02)$, with RSAS was $0.76 \pm 0.11(\mathrm{p}<0.02)$, with RR $0.87 \pm 0.11(\mathrm{p}<0.02)$, with $\mathrm{LF}_{\mathrm{SP}}-0.81 \pm 0.09(\mathrm{p}<0.02)$, and with $\mathrm{LF}_{\mathrm{RR}} / \mathrm{HF}_{\mathrm{RR}}$ ratio was $-0.77 \pm 0.13(\mathrm{p}<0.05)$.
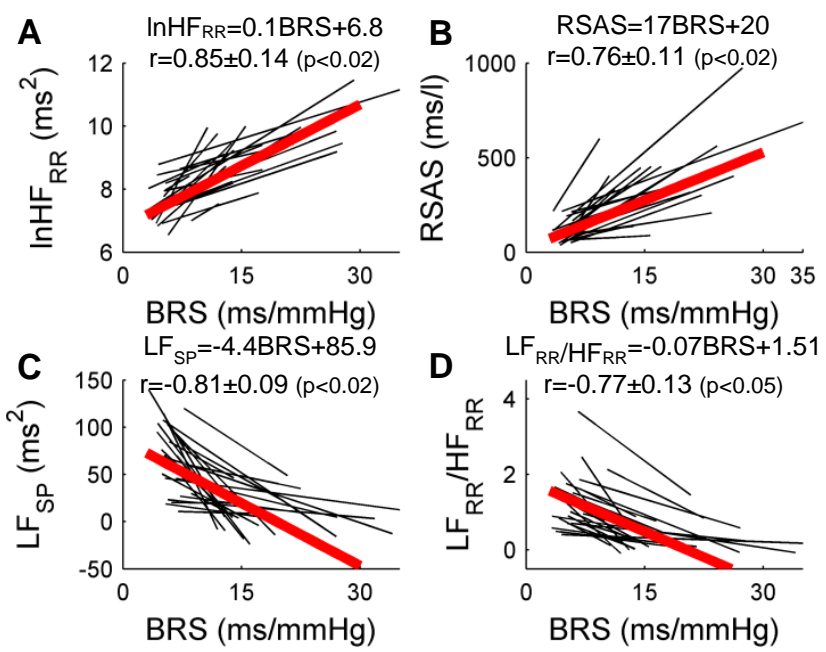

Fig. 3. Individual (thin black lines) and mean (thick grey lines) linear regressions of BRS vs. A) $\operatorname{lnHF}_{R R}, B$ ) RSAS, C) LFsp, D) $\mathrm{LF}_{\mathrm{RR}} / \mathrm{HF}_{\mathrm{RR}}$ and E) LFPp.

\section{Discussion}

Our results establish that, in healthy subjects, BRS, RSAS and sympathovagal balance measures are tightly related, on the basis of the opposite effects caused by CFT and AOT on these variables, assessed in a beat-tobeat format. Our main findings are that the mean changes of BRS, $\ln \mathrm{HF}_{\mathrm{RR}}$ power, RR and RSAS are at least two times larger in CFT than in AOT; that their instantaneous values present characteristic response patterns of overshoot in CFT, of sudden onset for $\operatorname{lnHF}_{\mathrm{RR}}$ and RSAS but gradual increase for BRS, and of undershoot of the four variables in AOT; and that BRS shows very strong correlations, direct with $\operatorname{lnHF}_{\mathrm{RR}}$, RSAS and RR, and inverse with $L F_{S P}$ power and the $L F_{R R} / H_{R R}$ ratio.

It has been documented that CFT increases vagal activity through trigeminal afferents, indicated by the rise of $\mathrm{HF}_{\mathrm{RR}}$ power [7] and decreased HR [6]. Also, that BRS and RSAS, both estimated by transfer function method, are increased during CFT [3]. In contrast with this static description, our findings suggest that CFT produces an immediate and sudden coactivation of vagal and sympathetic outflows but with a clear vagal predominance, as reflected by the resulting HR reduction (Fig. 2B). The sympathetic activation is responsible for 
the abrupt increase of PP (Fig. 2A). The increased BRS (Fig. 2C) during CFT is evidence of the baroreflex mechanism participation which, triggered by the PP increase, reinforces vagal activity and RR lengthening while minimizing sympathetic activity, as indicated by the $\mathrm{LF}_{\mathrm{RR}} / \mathrm{HF}_{\mathrm{RR}}$ ratio, which falls and remains below its baseline level throughout the maneuver (Fig. 2G). The increase of RSAS contributes to the increase of vagal activity (Fig. 2D and E). Therefore, the sympathovagal coactivation is followed by the involvement of baroreflex and RSAS mechanisms. Our explanation challenges the accepted concept that the sympathovagal coactivation produced by CFT is baroreflex-independent [6].

Few studies have reported, in an instantaneous format, the large fluctuations of HR [4], AP [5] and $\mathrm{HF}_{\mathrm{RR}}$ power [8] during the first minute of AOT, but the time course of BRS values has not been examined. Steady-state spectral analysis of cardiovascular variability has been performed after the first minute of the maneuver onset. In these conditions, it has been reported that the $\mathrm{LF}_{\mathrm{RR}} / \mathrm{HF}_{\mathrm{RR}}$ ratio increases and that both $\mathrm{HF}_{\mathrm{RR}}$ and BRS are reduced [2].

An explanation of our findings in AOT considers that the central command, which drives the voluntary movements required for standing up and then laying down, contributes to the abrupt and large initial sympathetic elevation mediated by the baroreflex mechanism, which consecutively decreases sympathetic outflow and then increases it again at the end of the maneuver (Fig. $2 \mathrm{C}$ and $\mathrm{G}$ ). The response pattern of vagal activity is the reciprocal of the sympathetic one (Fig. 2D). It is possible that the abrupt initial sympathetic increase and vagal withdrawal cause the marked reductions of BRS, RSAS and their respective coherences. These changes, by strengthening the former and sustaining the latter, ensure an efficient AP compensatory rise. As sympathetic activity decreases, BRS and RSAS gradually recover towards their control levels and show an overshoot in the recovery stage, associated with vagal predominance (Fig. 2). By pointing out the fall of vagal activity and the striking changes of BRS and RSAS from an undershoot to an overshoot, the above explanation extends the accepted notion that AOT provokes a baroreflex-dependent sympathetic response $[1,5,8]$.

CFT and AOT can be used in clinical and physiological settings to provoke opposite changes in BRS, vagal activity, RSAS, HR and PP, with changes at least two times larger in CFT than in AOT. Moreover, the strong correlations of BRS with RSAS, vagal indexes and sympathetic measures (Fig. 3), suggest a reciprocal mechanism with stereotyped autonomic-cardiovascular response. Thus, the maneuvers or corporal conditions associated to sympathetic activation, such as AOT, would reduce BRS and RSAS to strengthen sympathetic activity and, through reciprocal innervation, restrain vagal outflow. In contrast, vagal maneuvers, such as CFT, would increase BRS and RSAS to strengthen vagal activity and, through reciprocal innervation, reduce the sympathetic one. To the best of our knowledge, this is the first study to establish that CFT and AOT, during their highly fluctuating initial minute, provoke opposite instantaneous response patterns in BRS, RSAS and sympathovagal balance, which are strongly correlated.

In conclusion, in a beat-to-beat format, BRS, $l n \mathrm{HF}_{\mathrm{RR}}$, RSAS, $L_{R R} / H_{R R}$ and RR: exhibit great dynamism, show strong correlations between them, and follow distinctive opposite patterns in response to each maneuver. While in CFT the greater values of BRS and RSAS allow amplifying the vagal activity and minimizing the sympathetic outflow, in AOT the lower values of BRS and RSAS determine the opposite autonomic effects. The strong correlations, positive between BRS and vagal activity and negative between BRS and sympathetic activity, suggest that the dynamic adjustments of BRS, together with those of RSAS, reciprocally strengthen the activity of the most situationally active autonomic branch while inhibiting the activity of the other one.

\section{References}

[1] Ptaszynski P, Kaczmarek K, Klingenheben T, et al. Noninvasive assessment of autonomic cardiovascular activity in patients with inappropriate sinus tachycardia. Am J Cardiol 2013; 112:811-5.

[2] Carrasco-Sosa S, Gaitán-González M, González-Camarena R, Yáñez-Suárez O. Baroreflex sensitivity assessment and heart rate variability: relation to maneuver and technique. Eur J Appl Physiol 2005; 95:265-75.

[3] Stemper B, Hilz M, Rauhut U, Neundörfer B. Evaluation of cold face test bradycardia by means of spectral analysis. Clin Auton Res 2002; 12:78-83.

[4] Cybulski G, Niewiadomski W. Influence of age on the immediate heart rate response to the active orthostatic test. J Physiol Pharmacol 2003; 54:65-80.

[5] Bahjaoui-Bouhaddi M, Henriet M, Cappelle S, et al. Active standing and passive tilting similarly reduce the slope of spontaneous baroreflex in healthy subjects. Physiol Res 1998; 47:227-35.

[6] Khurana R, Wu R. The cold face test: a non-baroreflex mediated test of cardiac vagal function. Clin Auton Res 2006; 16:202-7.

[7] Kinoshita T, Nagata S, Baba R, Kohmoto T, Iwagaki S. Cold-water face immersion per se elicits cardiac parasympathetic activity. Circ J 2006; 70:773-6.

[8] Martinmäki K, Rusko H, Saalasti S, Kettunen J. Ability of short-time Fourier transform method to detect transient changes in vagal effects on hearts: a pharmacological blocking study. Am J Physiol 2006; 290:H2582-9.

Address for correspondence.

Salvador Carrasco-Sosa

Depto. Ciencias de la Salud, S-353

Universidad Autónoma Metropolitana-Iztapalapa.

Av. San Rafael Atlixco \# 186, C.P. 09340 CDMX, México.

scas@xanum.uam.mx. 